\title{
IMF variations in dwarf galaxies
}

\section{Weidner and P. Kroupa}

\author{
Observatory of the University of Bonn, Auf dem Hügel 71, D-53121 Bonn, Germany
} email: cweidner, pavel@astro.uni-bonn,de

\begin{abstract}
Dwarf galaxies tend to have a large spread in metal abundances and overall lower metallicites than large Spiral or Elliptical ones. Here we propose that if all stars are formed from an invariant canonical IMF within each star cluster which are distributed according to an embedded cluster mass function (ECMF), then the integrated stellar IMF over the whole galaxy (the integrated galaxial IMF, IGIMF) must be steeper than the canonical IMFs within the clusters, and additionally they must depended on the stellar mass of the galaxy. In particular, dwarf galaxies and low-surface brightness galaxies show steeper galaxial IMFs which vary strongly with the star-formation rate. The resulting number of Supernovae II per low-mass star, and the chemical enrichment history of these galaxies therefore vary substantially depending on the galaxy assembly history.
\end{abstract}

Keywords. galaxies: abundances, galaxies: dwarf, galaxies: luminosity function, mass function, galaxies: star clusters, galaxies: stellar content

\section{The stellar and the (embedded) cluster IMF}

Stars form in embedded clusters with a few to many million stars. Inside these clusters they appear to form following a universal initial mass function (IMF) with a Salpeter power-law slope $\left(\alpha_{3}=2.35\right.$, Salpeter 1955, Kroupa 2002) above $1 M_{\odot}, \xi(m) \propto m^{-\alpha_{i}}$, with $\alpha_{0}=0.30$ for $0.01 \leqslant m / M_{\odot} \leqslant 0.08, \alpha_{1}=1.30$ for $0.08 \leqslant m / M_{\odot} \leqslant 0.50, \alpha_{2}=2.30$ for $0.50 \leqslant m / M_{\odot} \leqslant 1.00$ and $\alpha_{3}=2.35$ for $1.00 \leqslant m / M_{\odot}$. This has been found to be the case for a wide range of different conditions in the MW, LMC and SMC and other galaxies.

Star clusters also seem to be distributed according to a (universal?) power-law embedded cluster mass function (ECMF), $\xi_{\text {ecl }} \propto M_{\text {ecl }}^{-\beta}$, where $M_{\text {ecl }}$ is the total mass in stars. We assume a single-slope power-law ECMF with $\beta=2.35$ between $5 M_{\odot}$ and $10^{6} M_{\odot}$.

\section{The integrated galaxial initial mass function (IGIMF)}

In our model all stars are born in clusters following a universal IMF but also clusters are formed from a universal ECMF. An important constrain is that the mass of a cluster limits the mass of the most massive star in it (Fig. 1a). The ECMF is connected to the star-formation rate of a galaxy via the following equation (Weidner, Kroupa \& Larsen 2004, Fig. 1b):

$$
\log \left(M_{\mathrm{ecl}, \max }\right)=\log \left(k_{\mathrm{ML}}\right)+(0.75 \cdot \log \mathrm{SFR})+6.77,
$$

where $k_{\mathrm{ML}}$ is the mass-to-light ratio, typically 0.0144 for young $(<6 \mathrm{Myr})$ clusters.

So the mass function for all stars born in all clusters, which we call the integrated galaxial initial mass function (IGIMF), becomes the following integral (Kroupa \& Weidner 2003),

$$
\xi_{\mathrm{IGIMF}}(m, t)=\int_{M_{\mathrm{ecl}, \min }}^{M_{\mathrm{ecl}, \max }(S F R(t))} \xi\left(m \leqslant m_{\mathrm{max}}\right) \xi_{\mathrm{ecl}}\left(M_{\mathrm{ecl}}\right) d M_{\mathrm{ecl}} .
$$



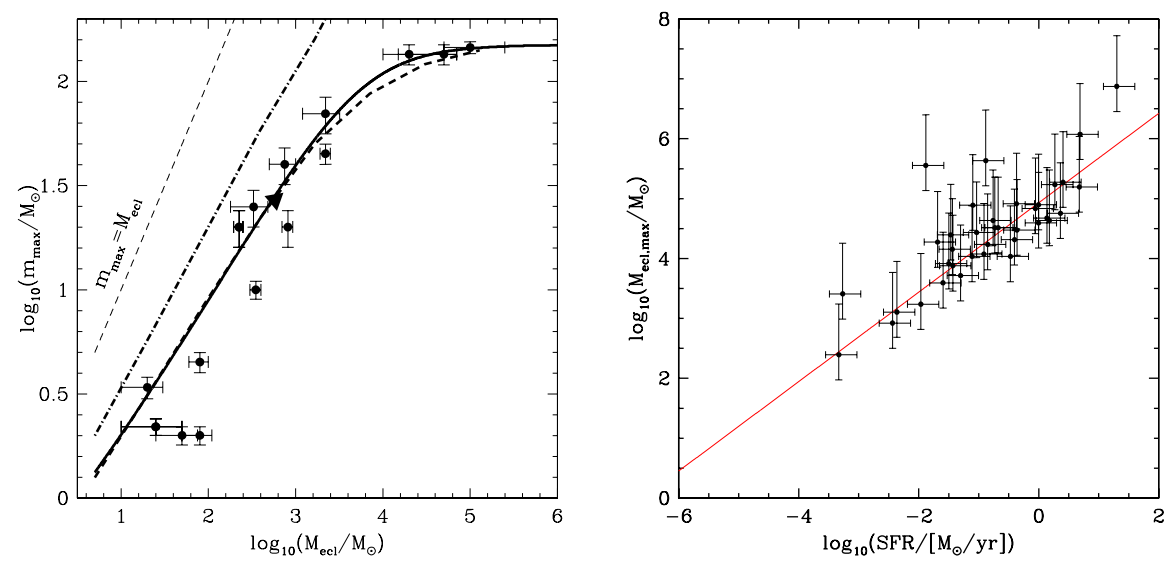

Figure 1. Left panel: The thick solid line shows the dependence of the maximum stellar mass on the cluster mass for $\alpha_{3}=2.35$ from the semi-analytical model (Weidner \& Kroupa 2004). The dashed line which follows close the semi-analytical result shows the mean maximum stellar mass for Monte-Carlo experiments when a fundamental upper mass limit of $m_{\max *}=150 M_{\odot}$ is invoked and the stars are sorted and then added until they reach the cluster mass $M_{\text {ecl }}$. The straight dashed line marks Monte-Carlo results without such a limit and for fully random sampling. The dots with error bars are observed clusters, while the triangle is a result from a star-formation simulation with an SPH code by Bonnell, Bate \& Vine (2003). Right panel: The solid line shows the star-formation-rate-maximal-cluster-mass relation from Weidner, Kroupa \& Larsen (2004) while the dots with the error bars are the observations.

As visible in Fig. 2a,b the resulting IGIMFs are always steeper than the input canonical stellar IMF.
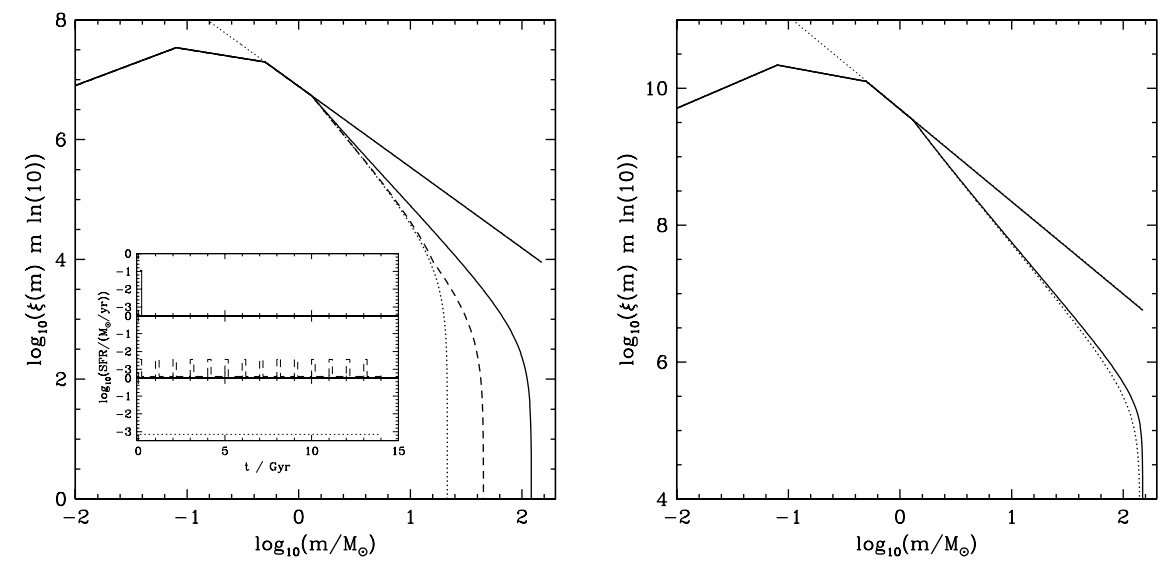

Figure 2. Left panel: Integrated galaxial mass functions for a galaxy with a stellar mass of $10^{7} M_{\odot}(\mathrm{dE}, \mathrm{dSph}, \mathrm{dIrr}, \mathrm{LSB})$ with different star-formation histories (solid curved line: single initial-burst of $100 \mathrm{Myr}$, dashed line: periodic SF of $100 \mathrm{My}$ every $900 \mathrm{Myr}$ and dotted line: constant SFR over 14 Gyr, respectively, from top to bottom) shown in the small box. The input IMF slope above $1 M_{\odot}$ is $\alpha_{3}=2.35$, while the ECMF slope $\beta$ is 2.35 . Right panel: The same for a galaxy with $10^{10} M_{\odot}$ stellar mass. Both taken from Weidner \& Kroupa (2005). 

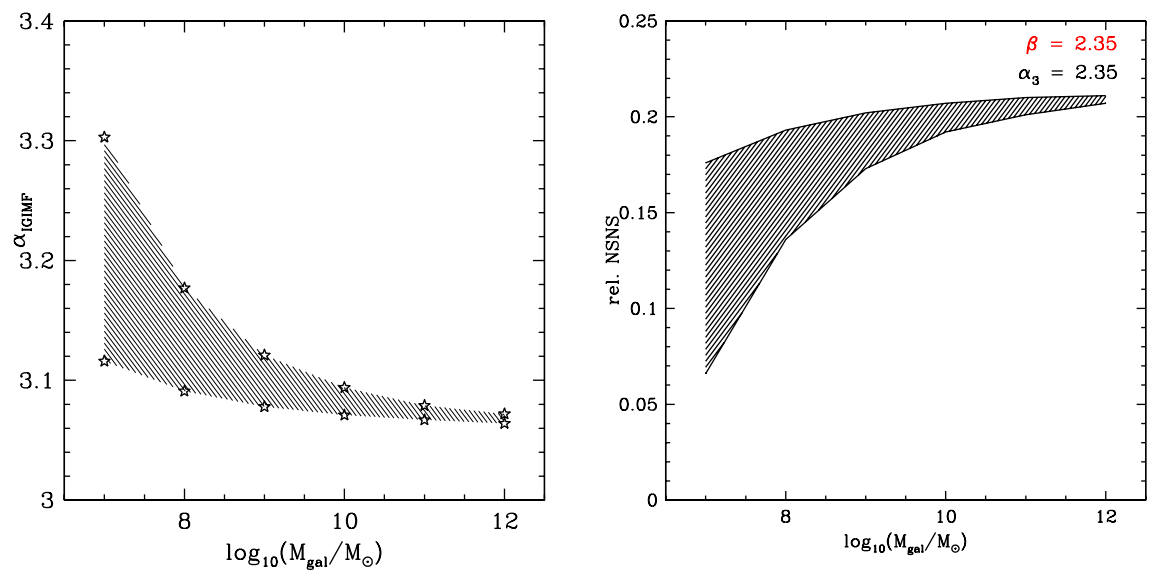

Figure 3. Left panel: IGIMF slopes above $\sim 1.3 M_{\odot}$ in dependence of the stellar galaxy mass for $\alpha_{3}=2.35$ and $\beta=2.35$. The lower limit of the shaded region results from single initial-burst models while the upper limit is from continuous models. The IGIMF thus becomes steeper with reducing stellar mass, thus resulting in systematic differences in the chemical evolution of different galaxy types. Also in the low-mass regime a larger scatter in chemical properties is to be expected. Right panel: The number of SNII per star relative to the same number for a constant canonical IMF as a function of galaxy mass for $\alpha_{3}=2.35$ and $\beta=2.35$. The upper limit of the shaded region results from single burst models while the lower limit is deduced from continuous star-formation models. While for galaxies with large stellar masses the value is about $20 \%$ of the corresponding canonical IMF value it drops significantilly for galaxies with lower stellar mass. Both panels are from Weidner \& Kroupa (2005).

\section{Results \& Conclusions}

- The IGIMFs must be steeper (Fig. 3a) than the stellar IMF and vary with galaxy type (Fig. 2).

- The number of SNII per star, the chemical enrichment histories and mass-to-light ratios calculated with an invariant Salpeter IMF may not be correct for any galaxy.

- The number of supernovae per star (Fig. 3b) is possibly significantly lower over cosmological times than for an invariant canonical IMF.

- Irrespective of how old a galaxy is it will always appear less chemically evolved than a more massive equally-old galaxy as a result of the steeper IGIMF (Fig. 4).

- The scatter in chemical properties must increase with decreasing galaxy mass.

\section{Acknowledgements}

This work has been funded by DFG grant KR1635/3.

\section{References}

Bonnell I.A., Bate M.R. \& Vine S.G. 2003 MNRAS 343, 413

Kroupa P. 2002, Science 295, 82

Kroupa P. \& Weidner C. 2003, ApJ 598, 1076

Salpeter E.E. 1955, ApJ 121, 161

Tremonti C.A., Heckman T.M., Kauffmann G., Brinchmann J., Charlot S., White S.D.M., Seibert M., Peng E.W., Schlegel D.J., Uomoto A., Fukugita M. \& Brinkmann J. 2004, ApJ 613, 898

Weidner C. \& Kroupa P. 2004, MNRAS 348, 187 


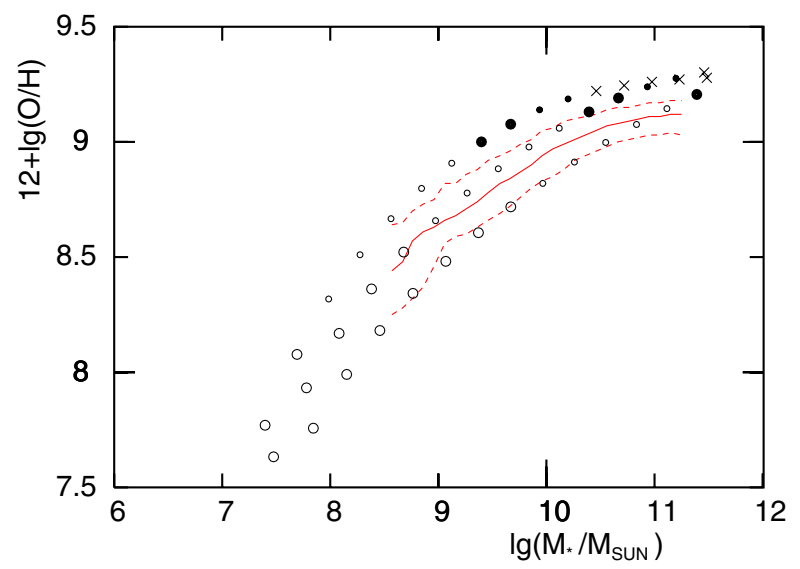

Figure 4. The relation between oxygen abundance and stellar mass of galaxies. Dashed lines and the solid line indicate the 16,50, and 84 percentiles of the observed metallicity distribution by Tremonti et al. (2004). The symbols are results from closed-box models and indicate the present-day gas fraction: large circle $>0.5$, small circle $0.5 \ldots 0.25$, large filled circle $0.25 \ldots 0.15$, small filled circle $0.15 \ldots 0.1$, and crosses $<0.1$.

Weidner C. \& Kroupa P. 2005, ApJ in press (astro-ph/0502525)

Weidner C., Kroupa P. \& Larsen S.S. 2004, MNRAS 350, 1503

\section{Discussion}

Gallagher: Can you indirectly test your model by predicting where observable massive stars, such as WR stars, will become rare due to IMF variations?

WEIDNER: Yes, one has to normalise the model on the number of observed unevolved stars and then calculate the expected number of WR and massive stars using stellar evolution models.

READ: How does your IMF for dwarf galaxies compare with direct observations e.g. Wyse et al. for the UMi dSph galaxy?

WEIDNER: The Wyse et al. results for masses above $1 M_{\odot}$ are also based on indirect constrains (abundances) and still subject to large uncertainties (e.g. yields). Other authors (e.g. Tolstoy et al. 2004, AJ, 125, 707) find for Sculptor, Fornax, Carina and Leo I dSph indications for an non-Salpeter IMF. We are in the progress of looking into the chemical evolution consequences of our model.

Hensler: The IGIMF slope for constant SF in e.g. dIrrs is much steeper then Salpeter. Does one significantly underestimate the past SFR if one applies a Salpeter slope with $\mathrm{M} / \mathrm{L}$ to observed luminosities? To what extent?

WEIDNER: There is a systematic error and we are looking into it. 